\title{
STABILIZING THE PERIODIC ORBITS IN A CHAOTIC MAPPING DESCRIBING THE DISCRETE HEALTH SYSTEMS VIA PREDICTION-BASED CONTROL
}

\author{
Dumitru Deleanu \\ Constanta Maritime University, Faculty of Naval Electro-Mechanics, 104 Mircea cel Batran Street, 900663, Constanta, \\ Romania, e-mail address: dumitrudeleanu@yahoo.com
}

\begin{abstract}
In the paper the problem of location and stabilization of unstable periodic orbits (UPOs) in discrete systems is investigated via the prediction-based control (PBC). It involves using the state of the free system one period ahead as reference for the control signal. Two types of control gains are tested, the first requiring the knowledge of the UPO to be stabilized and the second depending only on the actual state of the trajectory. The effectiveness of PBC is demonstrated on a chaotic mapping describing the malignant tumor growth. When the results obtained with the two control laws are compared with each other, it is found that the second variant is qualitatively superior, both in terms of convergence and the number of stabilized UPOs, especially for long-period orbits.
\end{abstract}

Key words: unstable periodic orbit, prediction - based control.

\section{INTRODUCTION}

In everyday life, human health is paramount for performing high-performance activities. some diseases can deteriorate the quality of work results.

Cancer, that terrible disease characterized by uncontrolled growth of cell and invasion into surrounding tissues, is one of the major causes of deaths in the world. It is natural, then, that the mathematical modelling approaches have become increasingly abundant in cancer research, with the ultimate goal of controlling it.

The mathematical models used to describe the growth rate of the cancer cells are either deterministic or stochastic. The widely used deterministic models are the exponential, logistic, Mendelsohn, Gompertz and Bartalanffy models. To provide best fits to the experimental and clinical data, some of the abovementioned models have been extended to their stochastic counterpart, e.g. Gompertz and logistic models [1 - 3]. Even if continuous models are usually used in the literature, some papers suggest that a suitable model for the tumor growth should be a discrete model, capable of exhibiting both periodic and complex chaotic dynamics $[4,5]$.

Generally, chaos is associated with unpredictable behaviour and this may be inconvenient in the treatment of the cancer tumor. For such a situation, suppression of chaos and its substitution with a regular behaviour may be achieved by control techniques [6,7]. Part of these approaches use the fact that the strange attractors associated to a chaotic dynamical system contain an infinite number of unstable periodic orbits (UPOs) forming the skeleton of the attractor. If such a UPO with low period is stabilized, it is expected that a tumor treatment action will be easier to identify and apply.

In the paper, we numerically investigated a technique proposed by Ushio and Yamamoto and developed by Chagas et al [8] for stabilizing UPOs in chaotic mappings, called prediction based control. As demonstrative example, we selected a one-dimensional map describing a malignant tumor growth.

\section{MODEL AND METHOD \\ 2.1 $\quad$ Model}

The one - dimensional discrete model used in the paper for describing a tumor growth was proposed by Ahmed and assumes that the cells of the tumor have a core and a surface and he cell growth is proportional to its surface while the loss with its volume [5]. The associated mapping, after rescaling, reads as:

$$
{ }_{n+1}=6.75 p\left(x_{n}^{2 / 3}-{ }_{n}\right) \triangleq f\left(x_{n}, p\right)
$$

where $x_{n}$ refers to the number of cancerous cells at "time" $n$, the parameter $p \in[0,1]$ indicates the tumor growth rate and the nonlinear function $f$ transforms the interval $[0,1]$ onto itself. The map has two fixed points, 


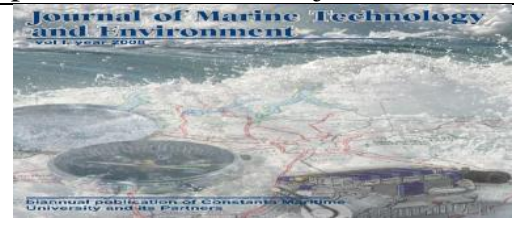

Journal of Marine technology and Environment ${ }^{-\prime}(p)=0$ and ${ }^{-\prime}(p)=\left(\frac{6.75 p}{1+6.75 p}\right)^{3}$. The second one is stable for $p \in(0.148,0.740)$ and then is replaced, through a pitchfork bifurcation, by a period- 2 orbit. This is, at its turn, stable for $p<\bar{p}_{c r}^{(2)}=0.898$ and then bifurcates into a 4 - cycle. The next bifurcation points are $\bar{p}_{c r}^{(4)}=0.924$ and $\bar{p}_{c r}^{(8)}=0.932$. Starting with an accumulation point, the map behaves chaotically, excepting some small windows with periodic behaviour.

\subsection{Method}

Consider the one-dimensional nonlinear map

$$
{ }_{n+1}=f\left(x_{n}, p\right), x_{n} \in R, n \geq 1
$$

where $p \in R$ is a parameter and $f \in C^{1}$.

Let $P^{(s)} \equiv\left({ }_{1}(p),{ }_{2}(p), \ldots, \bar{x}_{s}(p)\right)$ be a period $-s$ orbit of map (2), meaning

$$
\begin{gathered}
-_{2}=f\left(\bar{x}_{1}\right),{ }_{3}=f\left(\bar{x}_{2}\right), \ldots, \bar{x}_{1}=f\left(\bar{x}_{s}\right) \\
P^{(s)} \text { is a fixed point of map } f^{(s)}=\underbrace{f^{\circ} f^{\circ} \ldots \circ f}_{\text {stimes }} . \text { Let }
\end{gathered}
$$

$M=f^{\prime}\left(\bar{x}_{1}\right) \cdot f^{\prime}\left(\bar{x}_{2}\right) \cdot \ldots \cdot f^{\prime}\left(\bar{x}_{s}\right)$. If $|M|<1$, the orbit $P^{(s)}$ is stable, otherwise it is unstable. The prediction based control suggested in [8] for stabilizing the $s$ cycle $P^{(s)}$ in the case $|M|>1$ is given by:

$$
n+1=f\left(x_{n}, p\right)+u_{n}\left(x_{n}\right)
$$

where the control signal $u_{n}$ is defined by

$$
u_{n}\left(x_{n}\right)=K_{n} \cdot\left(f^{(s)}\left(x_{n}, p\right)-{ }_{n}\right)
$$

The control gain $K$ may be time varying or constant (that depends on the system state). In the paper we tested two variants of choosing $K$, namely:

$$
\text { V1) } K_{n}\left(\bar{x}_{i}\right)=\frac{f^{\prime}\left(\bar{x}_{i}\right)}{1-M}, i=\overline{1, s}
$$

$K_{n}$ is a time - periodic state - dependent gain and its determination depends upon unstable periodic orbit $P^{(s)}$ knowledge.

$$
\text { V2) } K_{n}\left(x_{n}\right)=\frac{f^{\prime}\left(x_{n}\right)}{f^{\prime}\left(x_{n}\right) \cdot f^{\prime}\left(\tilde{x}_{n+1}\right) \cdot \ldots \cdot f^{\prime}\left(\tilde{x}_{n+s-1}\right)}
$$

$K_{n}$ is a non-linear time - variant state - dependent gain, but its determination does not require any knowledge on the orbit $P^{(s)}$ (except the period $s$ ). The values $\tilde{}_{n+i}, i=$ $\overline{1, s-1}$ are computed along the trajectory of the free system (2).

\section{RESULTS}

Throughout this section, without exception, the parameter value $p=0.97$ is considered, for which the map (1) behaves chaotically. The strange attractors contains two fixed points, one orbit of period 2 and one of period 4, no orbits of period 3 and 5, two orbits of period 6 , and so on.
Year 2015, Vol.1

\subsection{Stabilizing the fixed point $(s=1)$}

For $p=0.97$, the nonzero fixed point is ${ }^{-\prime \prime}(0.97)=$ $0.652856 \triangleq x_{f}$, for which $M=-1.5159$. It can be stabilized in a small number of iterations both by law (6) and by law (7).
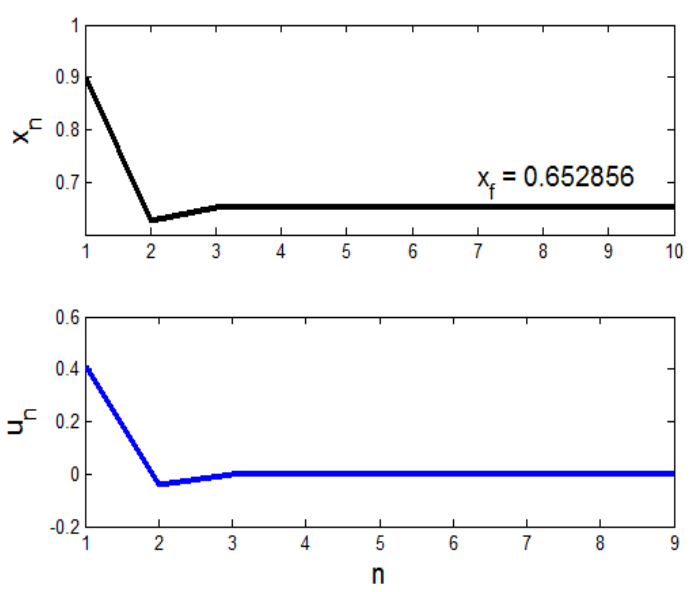

(a)

Figure 1 (a) Evolution of the state ${ }_{n}$ toward fixed point $f$ and the control effort $u_{n}$ for map (1) controlled by law (6) with ${ }_{1}=0.9$;
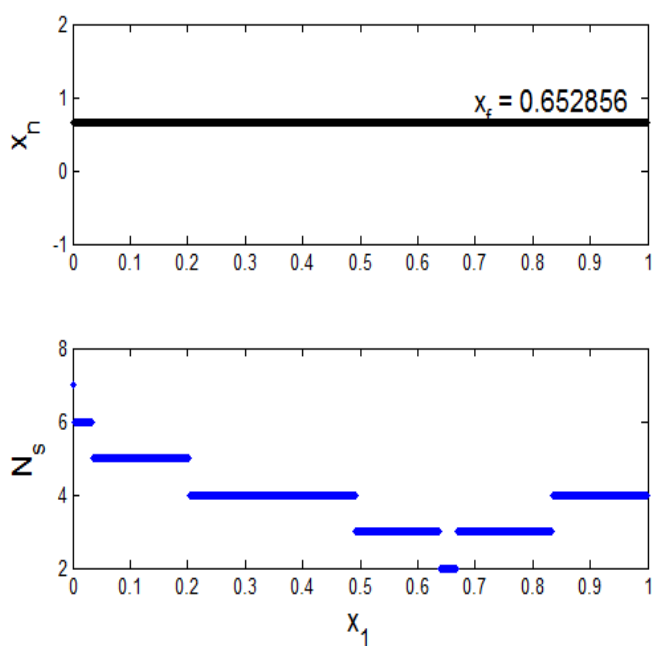

Figure $1(b)$ Basin of attraction of stabilized fixed point $f$ of map (1) controlled by law (6) and the number of iterations until stabilization, $N_{S}$; 
Journal of Marine technology and Environment
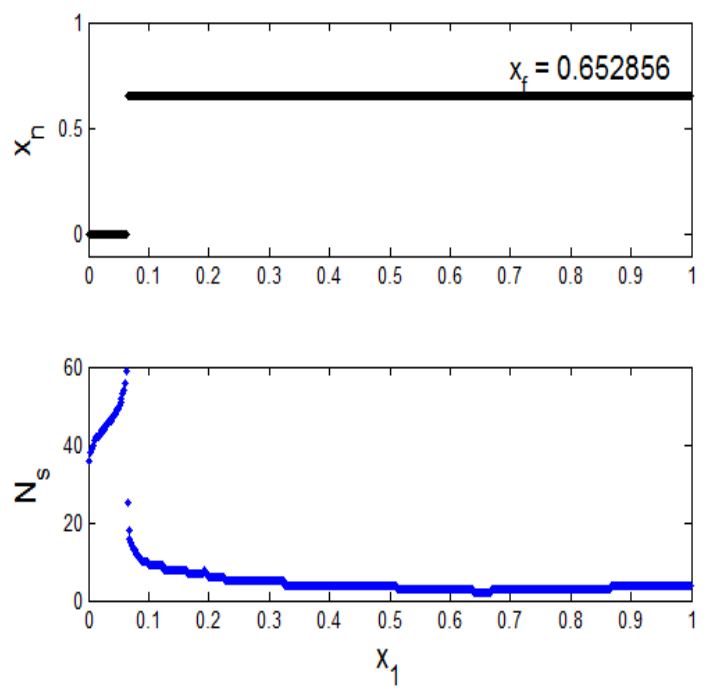

Figure $1(c)$ The same as in previous figure, but for law (7). In addition to $x_{f}$, there are initial conditions $x_{1}$ for which the zero fixed point is stabilized.

Figure 1 (a) shows the evolution of the state $n$ and the control effort $u_{n}$ for initial condition ${ }_{1}=0.9$ and law (6). After four iterations, the value $\left|u_{n}\right|<10^{-8}$ was reached, which we will choose as threshold for stabilization. In fact, the initial condition $x_{1}$ is not too important for the stabilization of $x_{f}$ under the conditions of law (6) enforcement. As indicated in Figure $1(b)$, the basin of attraction (BA) of $x_{f}$ is the entire interval $[0,1]$. The number of iterations until stabilization is between $N_{S}=2$, in the vicinity of the fixed point, and $N_{S}=6$, towards the left end of the interval. When applying the law (7), the situation is slightly different. The fact that the fixed point is not a priori known makes the choice of $x_{1}$ near the origin to produce the stabilization of the zero fixed point (in a much larger number of iterations). For the rest of the range $[0,1]$, there are no notable differences between the results provided by laws (6) and (7), as illustrated in Figure 1 (c). Moreover, this drawback of law (7) is maintained for all $p \in[0.74,1]$, as shown in Figure 2.

The white area refers to the zero fixed point and the red line to $x_{f}$.
Year 2015, Vol.1
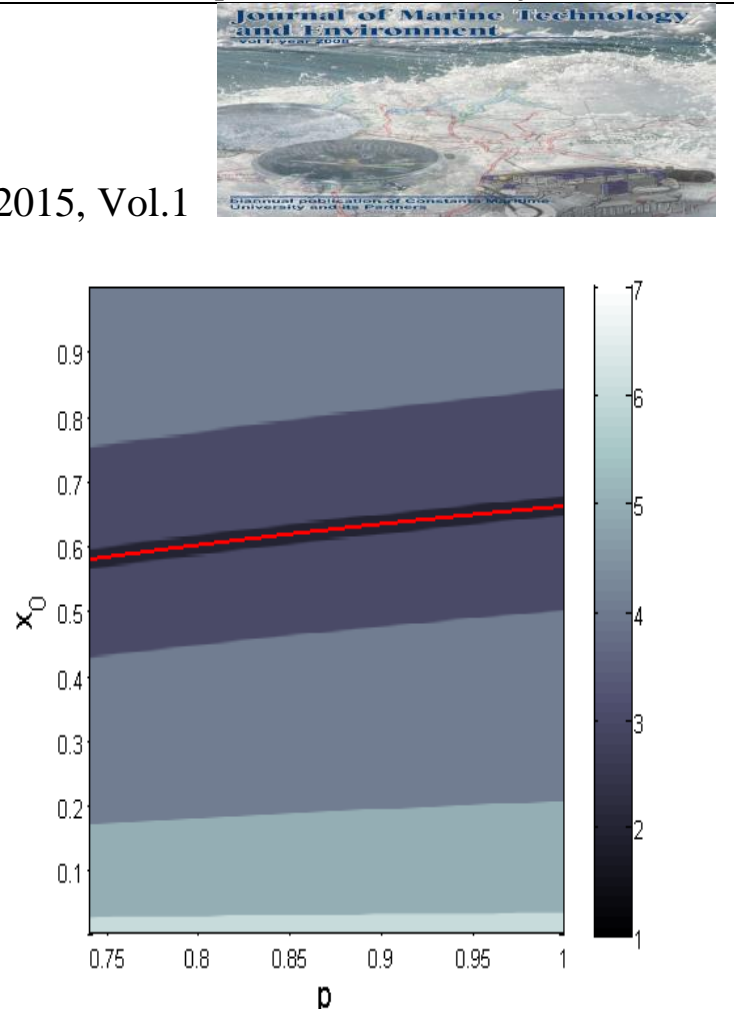

(a)

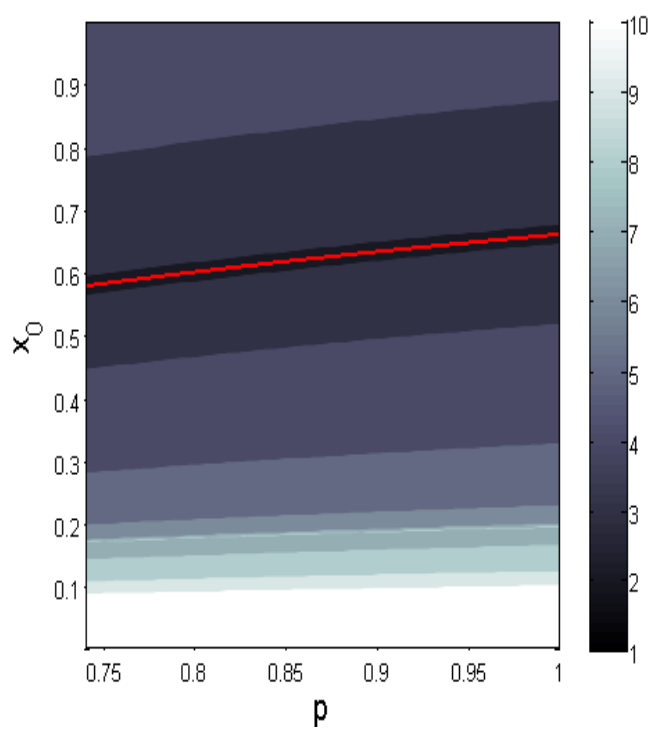

(b)

Figure 2 Dependence of the number of iterations until the fixed point's stabilization on the starting point and parameter $p$ when applying: (a) law (6) ; (b) law (7).

\subsection{Stabilizing the period -2 orbit $(s=2)$}

The only orbit of period 2 obtained for $p=0.97$ is ${ }_{1}=0.183799$ and ${ }_{2}=0.913170(M=-2.314003)$. In Figure 3, this orbit is stabilized for ${ }_{1}=0.9$ in ten iterations. The control gains are $K_{n}\left(\bar{x}_{1}\right)=0.340882$ and $K_{n}\left(\bar{x}_{2}\right)=-0.618090$. 
Journal of Marine technology and Environment
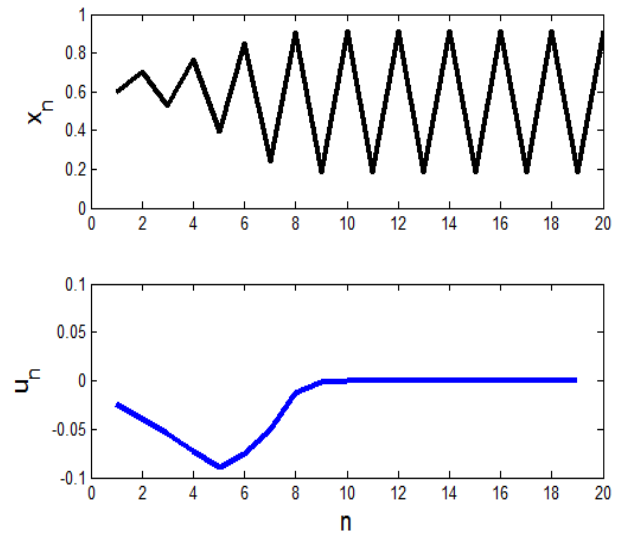

Figure 3 Stabilizing the period -2 orbit with the law (6)

The basin of attraction (BA) depends in a significant way on the connection between the parity of index " $n$ " in the control scheme (4) and the components of the orbit [9].
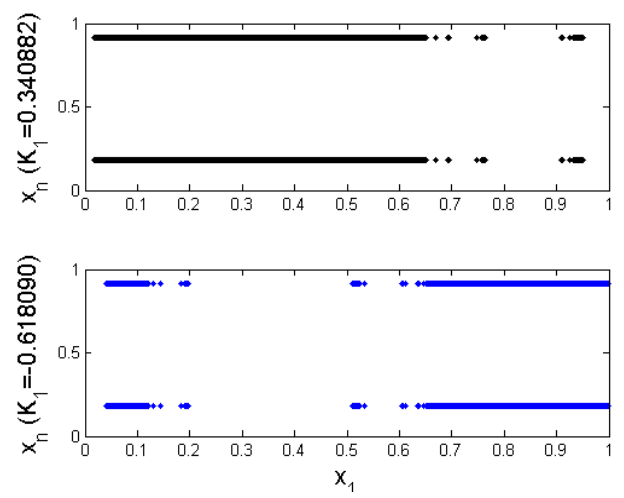

Figure 4 (a) Dependence of the basin of attraction of the period- 2 orbit on the choice of control gains in (6)
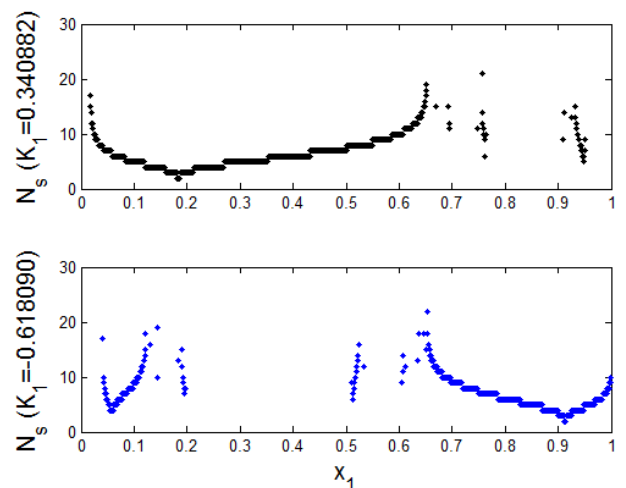

Figure 4(b) The same, but for the number $N_{S}$ of iterations until stabilization

If an odd " $n$ " corresponds to $\bar{x}_{1}$, then the BA is formed mainly in the closeness of this component (see the upper panel in Figure $4(a)$ ). Otherwise, the BA surrounds the component $\bar{x}_{2}$ (see lower panel in Figure 4 (a)). The number $N_{S}$ of steps until the orbit is stabilized

\section{Year 2015, Vol.1}

remains less than ten, except for the marginal areas of the BA, as shown in Figure $4(b)$.

Unlike variant V1, the application of law (7) makes that every time when the starting point does not lead to the stabilization of period -2 orbit, the non-zero fixed point $x_{f}$ is stabilized instead (see Figure 5).

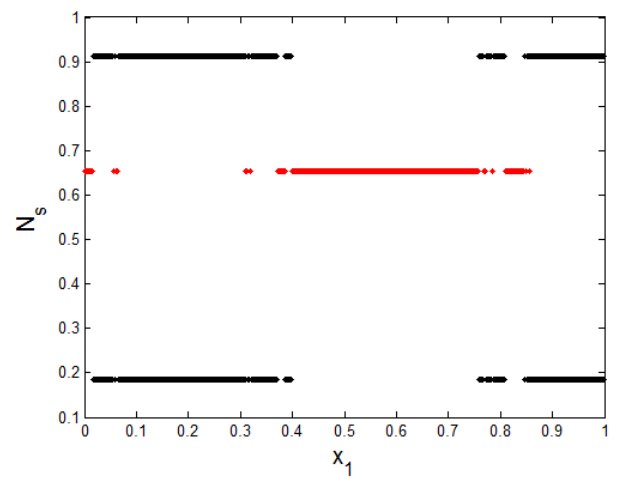

(a) (a) Basins of attraction;

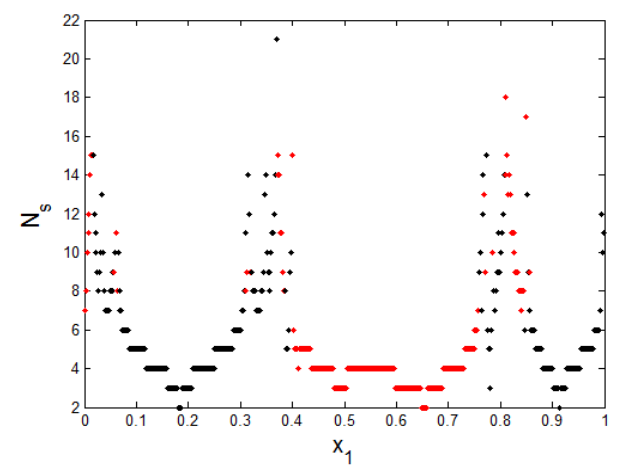

(b) Number of iterations required for stabilization. The red colour stands for $x_{f}$.

Figure 5 Stabilizing the period-2 orbit and the nonzero fixed point with the law (7):

\subsection{Stabilizing the period-6 orbit $(s=6)$}

For $p=0.97$, there exist two orbits of period 6, namely $P_{1}^{(6)}=\{0.09226,0.73277,0.52393,0.82481$, $0.35808,0.95710\}$ and $P_{2}^{(6)}=\{0.10520,0.77030$, $0.458379,0.891233,0.228372,0.950978\}$. The law (6) with gains $K_{n}\left(\bar{x}_{1}\right)=0.29190, K_{n}\left(\bar{x}_{2}\right)=-0.15997$, $K_{n}\left(\bar{x}_{3}\right)=-0.10625, K_{n}\left(\bar{x}_{4}\right)=-0.17723, K_{n}\left(\bar{x}_{5}\right)=$ $-0.03756, K_{n}\left(\bar{x}_{6}\right)=-0.19864 \quad$ and $\quad{ }_{1}=0.9$ succeeded to stabilize orbit $P_{1}^{(6)}$ in 12 iterations, as presented in Figure 6. 
Journal of Marine technology and Environment
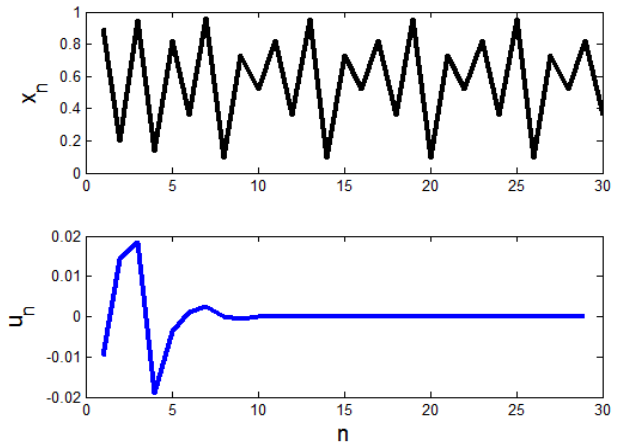

Figure 6 Stabilizing the period -6 orbit $P_{1}^{(6)}$ with the law (6).

When looking for the BA of this orbit, it is observed some initial conditions ${ }_{1}$ that lead to the period -2 orbit. Thus, out of the 1000 starting points 1,122 stabilized the period-2 orbit and 680 the orbit $P_{1}^{(6)}$ (see Figure $7(a)$ ). As for the number $N_{S}$, it fluctuates a lot with 1 and can reach values between 50 and 200 for many $x_{1}$ (see Figure $7(b)$ ).

At the scale of the representation in Figure $7(a)$ it seems that, for example, the whole interval $[0.3,0.4]$ is part of the BA for orbit $P_{1}^{(6)}$. A reduction of numerical investigation only at this interval, with a greater fragmentation of it, shows that there are, from place to place, points $x_{1}$ in the BA of period-2 orbir (see Figure 7 (c)).

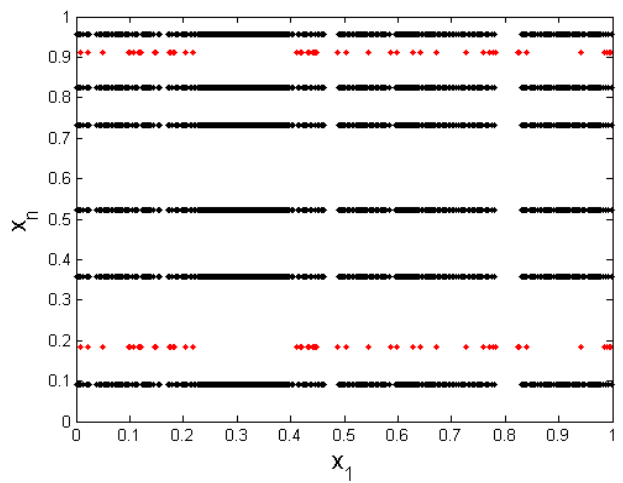

Figure 7 (a) Basins of attraction of the period- 6 orbit $P_{1}^{(6)}$ and period-2 orbit obtained by using law (6)

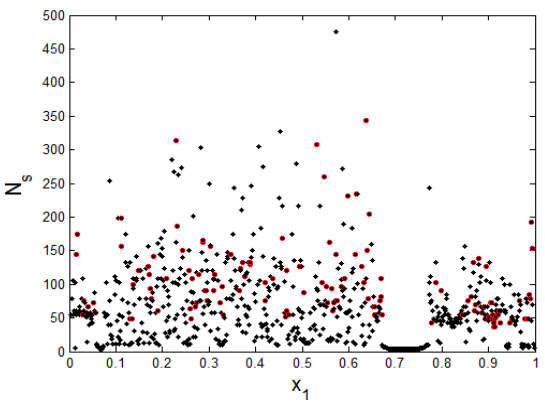

Figure $7(b)$ The associated number of iterations until stabilization. The red colour stands for the period-2 orbit
Year 2015, Vol.1

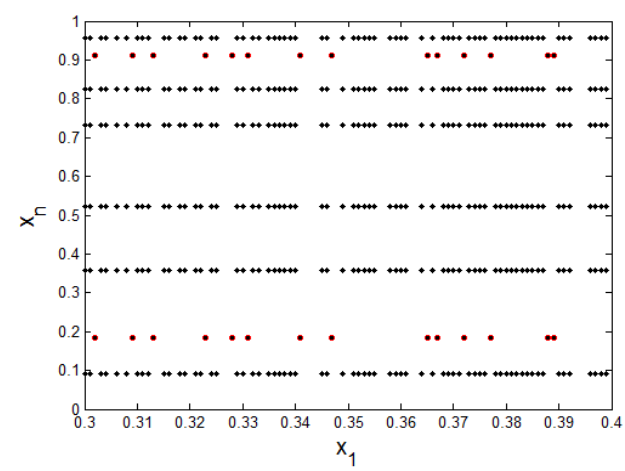

Figure $7(c)$ Detail in Figure $7(a)$

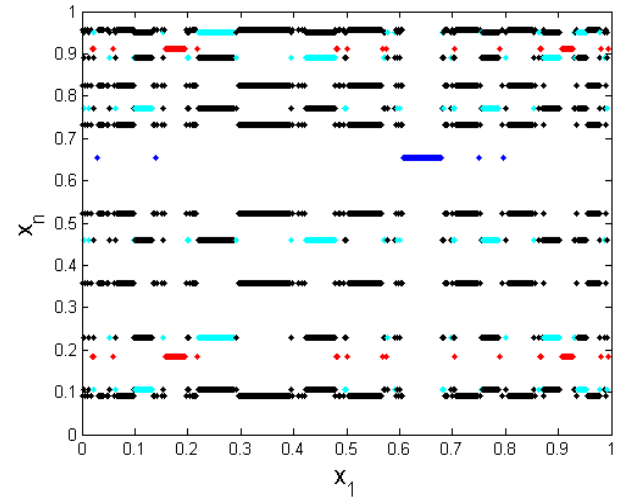

Figure 8 (a) Basins of attraction for the nonzero fixed point (blue colour), period-2 orbit (red), period - orbit $P_{1}^{(6)}$ (black) and period -6 orbit $P_{2}^{(6)}$ (cyan), obtained by using the law (7)

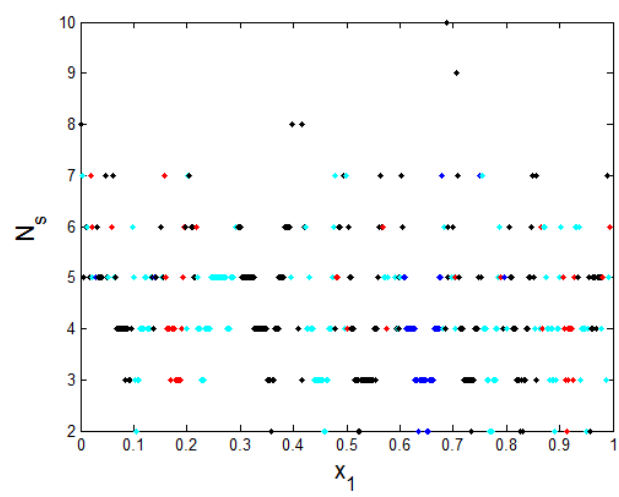

(b)

Figure $8(b)$ The associated number of iterations until stabilization

The law (7) begins to prove its superiority starting with $s=4$ both in terms of the speed of the stabilization process, and in the percentage of initial conditions $x_{1}$ leading to a stable orbit. All the orbits with period divisor of $s$ have a chance to be detected and stabilized.

Thus, for $s=6,446$ of the 500 starting points $x_{1}$ had, as a result of application of law (7), a stabilized 
Journal of Marine technology and Environment cycle: 41 found the nonzero fixed point, 46 the period- 2 orbit, 205 the orbit $P_{1}^{(6)}$ and 154 the orbit $P_{2}^{(6)}$. In addition, the number $N_{S}$ was much smaller than for the law (6), regardless of the stabilized orbit, as shown in Figure 8 .

\section{CONCLUSIONS}

In the paper we have numerically analyzed a chaos control technique called prediction-based control method. The simulation were carried out on a onedimensional mapping describing the malignant tumor growth. The main conclusions of our study can be summarized as follows:

i) The method allow to stabilize UPOs up to any period for a large set of initial conditions. Its algorithm is quite straightforward and easy to implement;

ii) There is great flexibility in choosing the control gains. These may depend on the prior knowledge of the orbit to be stabilized (which is a drawback) or can be obtained only on the basis of the actual state of the analyzed dynamical system. In the second variant, the PBC can be used for finding UPOs before using, eventually, other control methods. Moreover, this option is qualitatively superion in terms of convergence, especially for long-period orbits;

iii) When it is desired to stabilize one/all perios $-s$ orbits it is possible that by choosing different initial conditions to reach the stabilization of some orbits with period divisor of $s$. The associated basins of attraction are very fragmented and decrease in length for higher values of $s$.

\section{REFERENCES}

[1] Anyuc, Y. et al., 2019, A review of mathematical models for tumor dynamics and treatment resistance evolution of solid tumors, CPT Pharmacometrics Syst. Pharmacol. Vol. 8, pp. $720-737$.

[2] Tabassum, S. et al., 2019, Mathematical modelling of cancer growth process: A review, J. Phys. Conf. Ser., Vol. 1366, 012018.

[3] Liu, X., Li, Q., Pan, J., 2018, A deterministic and stochastic model for the system dynamics of tumorimmune responses to chemoterapy, Physica A: Statistical Mechanics and its Applications, Vol. 500, pp. 162 - 176.

[4] Virani, K., 2016, Discrete model for cancer tumor growth and effect of chemoterapy, $\mathrm{PhD}$ Thesis, City College of New York.

[5] Ahmed, E., 1993, Fractals and chaos in cancer models, Int. J. Theor. Phys., Vol. 32, pp. 353 - 355.

[6] Deleanu, D., Panaitescu, V.P., 2013, Controlling chaos in biological populations with non-overlapping
Year 2015, Vol.1 Jompnat of Marimo Tochmology

generations, Recent Advances in Circuits, Telecommunications and Control Conference (CCTC'13), Paris, France.

[7] Deleanu, D., Dumitrache, C.L., 2020, On predictive control stabilization of periodic orbits in a chaotic mapping describing tumor growth: A numerical analysis, The $8^{\text {th }}$ IEEE International Conference on EHealth and Bioengineering (EHB 2020), Iasi, October 29 -30 , Web Conference.

[8] Chagas, T., Bliman, P.A., Kienitz, K.H., 2018, Stabilization of periodic orbits of discrete-time dynamical systems using the Prediction-Based control: New control laws and practical aspects, Journal of the Franklin Institute, Elsevier, Vol. 355, no. 2, pp. 4771 4793.

[9] Deleanu D., 2018, “Suppressing chaos in population dynamics discrete models by adding periodic pulses to the system variables, Journal of Marine Technology and Environment, Vol.1, pp 15-22, Nautica Publishing House, Constanta, Romania. 